\title{
Finite-time Stabilization for a Class of Networked Systems with Delay
}

\author{
Hejun Yao*
}

School of Mathematics and Statistics, Anyang Normal University, Anyang, 455000, China

\begin{abstract}
The finite-time stabilization problem for a class of networked control systems with state delay is considered in this paper. With Lyapunov theorem, a sufficient condition for the design of a state feedback controller which makes the closed loop system finite-time stable is provided. And, the sufficient condition is given in terms of linear matrix inequality. A numerical example is presented to illustrate the proposed methodology.
\end{abstract}

Keywords: Networked control systems, delay, finite-time stabilization.

\section{INTRODUCTION}

Feedback control systems wherein the control loops are closed through a real-time network are called networked control systems (NCSs). The main feature of NCSs is that the components (sensors, controller and actuators) of the system are connected by a network. Compared with traditional point-to-point design, the NCSs have many advantages such as low cost, wiring reduction, simple installation and maintenance, and high reliability [1-3]. For these reasons, NCSs have been widely applied to many complicated control systems, such as aviation and aerospace fields $[4,5]$.

However, the insertion of the communication network in feedback control loop makes the analysis and design of NCSs complicated. The change of communication architecture induces different forms of time delay uncertainty between sensors, actuators and controllers. These time delays come from the time sharing of the communication medium as well as the computation time required for physical signal coding and communication processing [6-8]. It is well known that time delays can degrade a system's performance and even cause system instability. Therefore, the issues of stability analysis and designing controllers for NCSs have received much consideration for decades [7-12].

Much work has been done on the robust control of NCSs over the past ten years. Most of the results in this field relate to stability and performance criteria defined over an infinite time interval. However, the main concern in many practical applications is the behavior of the dynamical systems over a fixed finite time interval [13], for example, large values of the state are not acceptable in the presence of saturations. Therefore, we need to check the unacceptable values that the system state does not exceed a certain threshold during a fixed finite-time interval by giving some initial conditions. The concept of finite-time stability referring to these transient performances of control dynamics dates back to the
Sixties, when it was introduced in the control literature [14]. Then, some attempts on finite-time stability can be found by using Lyapunov functional approach [15]. Recently, with the aid of LMIs techniques, more concepts of finite-time stability have been proposed for linear continuous-time or discrete-time control system in the literatures; see, for instance [16-22].

But the above papers consider the Lyapunov stability for NCSs, a few results on finite-time stability for NCSs has been reported. Based on this fact, some new methods and approaches should be developed for designing controllers for NCSs, which motivates this paper. Inspired by the above literature, in this paper, the attention is focused on the finitetime stabilization of a class of NCSs with state delay and communication delays. The design is divided in two steps: the synthesis condition of the state feedback controller, supposing that the state variables are available, and then the sufficient condition is given in terms of an LMI optimization problem. Conversely, the approach proposed in this paper leads to an LMI formulation, which gives the opportunity of fitting the finite-time control problem in the general framework of the LMI approach to the multi-objective synthesis.

This paper is organized as follows: in Section 2 the definition of finite-time stability is recalled and the problem we want to solve is formally stated. In section 3 , we address the finite-time stability problems, namely sufficient conditions for the existence of state feedback controllers guaranteeing finite-time stabilization of the closed loop systems are provided. In section4, it is shown how the proposed design condition can be expressed in terms of LMIs and, therefore, can be efficiently dealt with, and a numerical example is provided. Some conclusions are drawn in Section 5.

\section{PRELIMINARIES}

In this paper we consider the following typical NCSs with state delay as shown in Fig. (1).

$$
\dot{x}(t)=A x(t)+A_{h} x(t-h)+B u(t)+B_{1} \omega(t)
$$




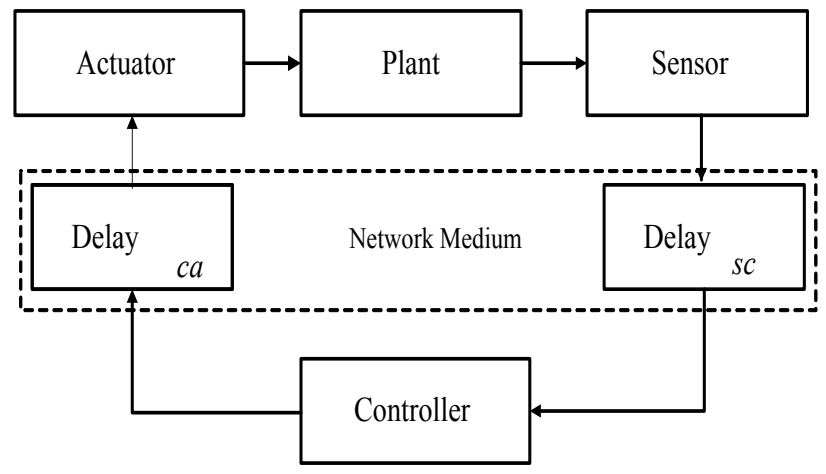

Fig. (1). A typical networked control system.

Where, $A, A_{h}, B, B_{1}$ take value in $R^{n \times n}, R^{n \times n}, R^{n \times m}, R^{n \times l}$, respectively. $x(t) \in R^{n}$ is the state vector, $u(t) \in R^{m}$ is the control input, and $h$ denotes system state delay. $\omega(t) \in R^{l}$ is the exogenous disturbance and satisfies.

$$
\int_{0}^{T} \omega^{T}(t) \omega(t) d t \leq d, d \geq 0
$$

To simplify the analysis, based on actual engineering background, a full characterization of this NCS is given by the following assumption.

Assumption 1. The sensor is time driven; the controller and actuator are event driven. We use $\tau_{s c}$ and $\tau_{c a}$ to represent the sensor-controller and controller-actuator delay, respectively, then the communication delay is given by $\tau=\tau_{s c}+\tau_{c a}$.

Considering the effect of communication delay $\tau$, the above plant model is transformed into an NCS model

$\dot{x}(t)=A x(t)+A_{h} x(t-h)+B u(t-\tau)+B_{1} \omega(t)$

Concerning NCSs (3), we design a state feedback controller

$u(t)=K x(t)$

Where, $K$ is a state feedback gain matrix to be determined later. Then, the resulting closed-loop NCSs follows that

$\dot{x}(t)=A x(t)+A_{h} x(t-h)+B K x(t-\tau)+B_{1} \omega(t)$

The aim of this paper is to find some sufficient conditions which guarantee the existence of a state feedback controller which stabilizes the system (1) over the finite interval $[0, T]$. By selecting the appropriate Lyapunov-Krasovskii function, the main results will be given in the form of LMIs. The general idea of finite-time control can be formalized through the following definitions over a finite-time interval for some given initial conditions [17-22].

Definition 1 (Finite-time stability). Given three positive scalars $c_{1}, c_{2}, T$, with $c_{1}<c_{2}$ and a positive matrix $R$, the time delay NCSs (3) (setting $\omega(t) \equiv 0)$ is said to be finitetime stable (FTS) with respect to $\left(c_{1}, c_{2}, T, R\right)$, if

$$
x^{T}(0) R x(0) \leq c_{1} \Rightarrow x^{T}(t) R x(t)<c_{2} \quad \forall t \in[0, T]
$$

Remark 1. Different with the concept of Lyapunov asymptotic stability, FTS is a practical concept used to study the behavior of the system within a finite interval. A system is said to be finite-time stable if, once we fix a finite-time interval, its state remains within prescribed bounds during this time interval. Obviously, a system which is FTS may be not Lyapunov asymptotically stable; conversely a Lyapunov asymptotically stable system could not be FTS if, during the transients state, its state exceeds the prescribed bounds.

Definition 2 (Finite-time boundedness). Given three positive scalars $c_{1}, c_{2}, T$ with $c_{1}<c_{2}$, a positive definite matrix $R$ and a class of signals $u(t)$, the time delay NCSs (3) is said to be FTB with respect to $\left(c_{1}, c_{2}, u, T, R, d\right)$, if condition (6) holds for all $u(t) \in R^{m}$.

Definition 3 (Finite-time stabilization via state feedback). Given three positive scalars $c_{1}, c_{2}, T$ with $c_{1}<c_{2}$, a positive definite matrix $R$, the time delay NCSs (3) is FTB with respect to $\left(c_{1}, c_{2}, T, R, d\right)$ if there exists a state feedback controller in the form (4) and the condition (6) holds.

Remark 2. Notice that conditions (7) can be guaranteed by letting $\omega^{T}(t) \omega(t) \leq d$ (see [17-19, 23-24]). In the presence of external inputs, FTS can lead to the concept of FTB. Thus, FTS can be recovered as a particular case of FTB by setting $d=0$, that is, FTB implies finite-time stability, but the converse is not the fact. FTB and FTS are open-loop concepts. The finite-time control problem concerns the design of a state feedback controller which ensures the FTS or the FTB of the closed-loop time-delay NCSs (3).

\section{Lemma 1 [3] The LMI}

$$
\left[\begin{array}{cc}
Y(x) & W(x) \\
W^{T}(x) & R(x)
\end{array}\right]>0
$$

Is equivalent to,

$$
R(x)>0, Y(x)-W(x) R^{-1}(x) W^{T}(x)>0
$$

Where, $Y(x)=Y^{T}(x), R(x)=R^{T}(x)$ and $W(x)$ depend on $x$.

\section{MAIN RESULTS}

In this section, we consider the finite-time control synthesis for NCSs with state delay and communication delay, in terms of LMIs, we obtain the sufficient condition for the finite-time stabilization via state feedback.

Theorem 1. Given three positive scalars $c_{1}, c_{2}, T$, with $c_{1}<c_{2}$, a positive definite matrix $R$, the time delay NCSs 
(3) is finite-time stabilization via state feedback with respect to $\left(c_{1}, c_{2}, T, R, d\right)$, if there exist a scalar $\alpha \geq 0$, positive definite matrices $P \in R^{n \times n}, Q \in R^{n \times n}, T \in R^{n \times n}, S \in R^{l \times l}$, and matrix $K \in R^{m \times n}$ such that the following matrix inequalities hold

$\left[\begin{array}{cccc}\Xi & P A_{h} & P B K & P B_{1} \\ * & -Q & 0 & 0 \\ * & * & -T & 0 \\ * & * & * & -\alpha S\end{array}\right]<0$

And,

$\frac{c_{1}\left(\lambda_{\max }(\tilde{P})+h \lambda_{\max }(\tilde{Q})+\tau \lambda_{\max }(\tilde{T})\right)+d \lambda_{\max }(S)\left(1-e^{-\alpha T}\right)}{\lambda_{\min }(\tilde{P})}<c_{2} e^{-\alpha T}$

Where,

$\Xi=P A+A^{T} P+Q+T-\alpha P, \tilde{P}=R^{-1 / 2} P R^{-1 / 2}$,

$\tilde{Q}=R^{-1 / 2} Q R^{-1 / 2}, \tilde{T}=R^{-1 / 2} T R^{-1 / 2}$ and $\lambda_{\max }(\bullet)$ and $\lambda_{\min }(\bullet)$ indicate the maximum and minimum eigenvalue of the augment, respectively.

Proof; For given symmetric positive definite matrix $P, Q, T$, we construct the following Lyapunov-Krasovskii function:

$V(x(t))=x^{T}(t) P x(t)+\int_{t-h}^{t} x^{T}(\theta) Q x(\theta) d \theta$

$+\int_{t-\tau}^{t} x^{T}(\theta) T x(\theta) d \theta$

The time derivative of $V(x(t))$ along the trajectories of system (5) is given by

$$
\begin{aligned}
\dot{V}(x(t))= & x^{T}(t)\left(P A+A^{T} P\right) x(t)+2 x^{T}(t) P A_{h} x(t-h) \\
& +2 x^{T}(t) P B K x(t-\tau)+2 x^{T}(t) P B_{1} \omega(t) \\
& +x^{T}(t) Q x(t)+x^{T}(t-h) Q x(t-h) \\
& +x^{T}(t) T x(t)+x^{T}(t-\tau) T x(t-\tau) \\
= & x^{T}(t)\left(P A+A^{T} P+Q+T\right) x(t) \\
& +2 x^{T}(t) P A_{h} x(t-h)+2 x^{T}(t) P B K x(t-\tau) \\
& +2 x^{T}(t) P B_{1} \omega(t)+x^{T}(t-h) Q x(t-h) \\
& +x^{T}(t-\tau) T x(t-\tau) \\
= & {\left[\begin{array}{c}
x(t) \\
x(t-h) \\
x(t-\tau) \\
\omega(t)
\end{array}\right]\left[\begin{array}{c}
x(t) \\
x(t-h) \\
x(t-\tau) \\
\omega(t)
\end{array}\right] }
\end{aligned}
$$

Where,

$$
\Pi=\left[\begin{array}{cccc}
P A+A^{T} P+Q+T & P A_{h} & P B K & P B_{1} \\
* & -Q & 0 & 0 \\
* & * & -T & 0 \\
* & * & * & 0
\end{array}\right]
$$

From condition (8), we have

$$
\begin{aligned}
\dot{V}(x(t)) & <\alpha x^{T}(t) P x(t)+\alpha \omega^{T}(t) S \omega(t) \\
& <\alpha V(x(t))+\alpha \omega^{T}(t) S \omega(t)
\end{aligned}
$$

Multiplying (11) by $e^{-\alpha t}$, we can obtain

$e^{-\alpha t} \dot{V}(x(t))-e^{-\alpha t} \alpha V(x(t))<\alpha e^{-\alpha t} \omega^{T}(t) S \omega(t)$

Furthermore

$\frac{d}{d t}\left(e^{-\alpha t} V(x(t))\right)<\alpha e^{-\alpha t} \omega^{T}(t) S \omega(t)$

By integrating the above inequality from 0 to $t$, with $t \in[0, T]$, it follows that

$e^{-\alpha t} V(x(t))-V(x(0))<\int_{0}^{t} \alpha e^{-\alpha \theta} \omega^{T}(\theta) S \omega(\theta) d \theta$

Noting that $\alpha \geq 0, \tilde{P}=R^{-1 / 2} P R^{-1 / 2}, \tilde{Q}=R^{-1 / 2} Q R^{-1 / 2}$, and $\tilde{T}=R^{-1 / 2} T R^{-1 / 2}$, we can obtain the following relation:

$x^{T}(t) P x(t) \leq V(x(t))$

$$
\begin{aligned}
& <e^{\alpha t} V(x(0))+\alpha d \lambda_{\max }(S) e^{\alpha t} \int_{0}^{t} e^{-\alpha \theta} d \theta \\
& <e^{\alpha t}\left[x^{T}(0) P x(0)+\int_{-h}^{0} x^{T}(\theta) Q x(\theta) d \theta\right. \\
& \\
& \left.+\int_{-\tau}^{0} x^{T}(\theta) T x(\theta) d \theta+d \lambda_{\max }(S)\left(1-e^{-\alpha t}\right)\right] \\
& <e^{\alpha t}\left[x^{T}(0) R^{1 / 2} \tilde{P} R^{1 / 2} x(0)\right. \\
& +\int_{-h}^{0} x^{T}(\theta) R^{1 / 2} \tilde{Q} R^{1 / 2} x(\theta) d \theta \\
& +\int_{-\tau}^{0} x^{T}(\theta) R^{1 / 2} \tilde{T} R^{1 / 2} x(\theta) d \theta \\
& \left.+d \lambda_{\max }(S)\left(1-e^{-\alpha t}\right)\right] \\
& <e^{\alpha t}\left[\lambda_{\max }(\tilde{P}) x^{T}(0) R x(0)\right. \\
& +\lambda_{\max }(\tilde{Q}) \int_{-h}^{0} x^{T}(\theta) R x(\theta) d \theta \\
& +\lambda_{\max }(\tilde{T}) \int_{-\tau}^{0} x^{T}(\theta) R x(\theta) d \theta \\
& \left.+d \lambda_{\max }(S)\left(1-e^{-\alpha t}\right)\right] \\
& <e^{\alpha T}\left[c_{1}\left(\lambda_{\max }(\tilde{P})+h \lambda_{\max }(\tilde{Q})+\tau \lambda_{\max }(\tilde{T})\right)\right. \\
& \left.+d \lambda_{\max }(S)\left(1-e^{-\alpha t}\right)\right]
\end{aligned}
$$

On the other hand, it yields

$$
x^{T}(t) P x(t)=x^{T}(t) R^{1 / 2} \tilde{P} R^{1 / 2} x(t) \geq \lambda_{\text {min }}(\tilde{P}) x^{T}(t) R x(t)
$$


Putting together (13) and (14) we have

$x^{T}(t) R x(t)$

$e^{\alpha T}\left[c_{1}\left(\lambda_{\max }(\tilde{P})+h \lambda_{\text {max }}(\tilde{Q})+\tau \lambda_{\max }(\tilde{T})\right)+\right.$

$<\frac{\left.d \lambda_{\max }(S)\left(1-e^{-\alpha T}\right)\right]}{\lambda_{\min }(\tilde{P})}$

Condition (9) and inequality (15) imply,

$x^{T}(t) R x(t) \leq c_{2}, \forall t \in[0, T]$.

This completes the proof. Therefore, the proof follows.

Theorem 2. Given three positive scalars $c_{1}, c_{2}, T$, with $c_{1}<c_{2}$, a positive definite matrix $R$, the time delay NCSs (3) is finite-time stabilization via state feedback $u(t)=\bar{K} X^{-1} x(t)$ with respect to $\left(c_{1}, c_{2}, T, R, d\right)$ if, there exist scalars $\alpha \geq 0, \lambda_{i}>0, i=1,2,3,4$, positive definite matrices $X \in R^{n \times n}, \bar{Q} \in R^{n \times n}, \bar{T} \in R^{n \times n}, S \in R^{l \times l}$, and matrix $\bar{K} \in R^{m \times n}$ such that the following matrix inequalities hold:

$\left[\begin{array}{cccc}\Theta & A_{h} X & B \bar{K} & B_{1} \\ * & -\bar{Q} & 0 & 0 \\ * & * & -\bar{T} & 0 \\ * & * & * & -\alpha S\end{array}\right]<0$

$\lambda_{1} R^{-1}<X<R^{-1}$

$\lambda_{2} \bar{Q}<\lambda_{1} X$

$\lambda_{3} \bar{T}<\lambda_{1} X$

$0<S<\lambda_{4} I$

$$
\left[\begin{array}{cccc}
d \lambda_{4}\left(1-e^{-\alpha T}\right)-c_{2} e^{-\alpha T} & \sqrt{c_{1}} & \sqrt{h} & \sqrt{\tau} \\
* & -\lambda_{1} & 0 & 0 \\
* & * & -\lambda_{2} & 0 \\
* & * & * & -\lambda_{3}
\end{array}\right]<0
$$

Where

$\Theta=A X+X A^{T}+\bar{Q}+\bar{T}-\alpha X$

Proof. Now we prove that the inequality (8) is equivalent to the inequality (16).

Pre-and post-multiplying the inequality (8) by blockdiagonal matrix $\operatorname{diag}\left\{P^{-1}, P^{-1}, P^{-1}, I\right\}$, we know the inequality $(8)$ is equivalent to

$$
\left[\begin{array}{cccc}
\Sigma & A_{h} P^{-1} & B K P^{-1} & B_{1} \\
* & -P^{-1} Q P^{-1} & 0 & 0 \\
* & * & -P^{-1} T P^{-1} & 0 \\
* & * & * & -\alpha S
\end{array}\right]<0
$$

Where,

$\Sigma=A P^{-1}+P^{-1} A^{T}+P^{-1} Q P^{-1}+P^{-1} T P^{-1}-\alpha P^{-1}$

By letting $X=P^{-1}, \bar{K}=K P^{-1}, \bar{Q}=P^{-1} Q P^{-1}, \bar{T}=P^{-1} T P^{-1}$, the inequality (22) is equivalent to inequality (16).

On the other hand, we denote

$\tilde{X}=R^{-1 / 2} X R^{-1 / 2}, \tilde{Q}=R^{-1 / 2} Q R^{-1 / 2}, \tilde{T}=R^{-1 / 2} T R^{-1 / 2}$

Consider that $R$ is the positive-definite matrix and

$\lambda_{\text {max }}(X)=\frac{1}{\lambda_{\text {min }}(P)}$

Now inequalities (17-20) imply that

$$
\begin{aligned}
& 1<\lambda_{\text {min }}(\tilde{P}), \lambda_{\text {max }}(\tilde{P})<\frac{1}{\lambda_{1}}, \lambda_{\text {max }}(\tilde{Q})<\frac{\lambda_{1}}{\lambda_{2}} \lambda_{\text {max }}(\tilde{P}), \\
& \lambda_{\text {max }}(\tilde{T})<\frac{\lambda_{1}}{\lambda_{3}} \lambda_{\text {max }}(\tilde{P}), \lambda_{\text {max }}(S)<\lambda_{4}
\end{aligned}
$$

With the Schur Lemma1, we know the inequality (21) is equivalent to

$d \lambda_{4}\left(1-e^{-\alpha T}\right)-c_{2} e^{-\alpha T}+\frac{c_{1}}{\lambda_{1}}+\frac{h}{\lambda_{2}}+\frac{\tau}{\lambda_{3}}<0$

With (23), the condition (9) follows that

$$
\begin{aligned}
& \frac{c_{1}\left(\lambda_{\text {max }}(\tilde{P})+h \lambda_{\text {max }}(\tilde{Q})+\tau \lambda_{\text {max }}(\tilde{T})\right)+d \lambda_{\text {max }}(S)\left(1-e^{-\alpha T}\right)}{\lambda_{\text {min }}(\tilde{P})} \\
& <d \lambda_{4}\left(1-e^{-\alpha T}\right)+\frac{c_{1}}{\lambda_{1}}+\frac{h}{\lambda_{2}}+\frac{\tau}{\lambda_{3}}
\end{aligned}
$$

Inserting the inequality (24) into (25), the inequality (9) is satisfied. This completes the proof.

Remark 3. If condition (16) in Theorem 2 is satisfied with $\alpha=0$, then system (3) is also asymptotically stable in the sense of Lyapunov. Moreover in this case the finite-time properties are guaranteed for all $T>0$.

Remark 4. We can see that the conditions in Theorem 1 and Theorem 2 are not LMIs with respective to $\alpha, \mathrm{c}_{2}$, since $\alpha, \mathrm{c}_{2}$ appear in a nonlinear fashion. However, once we fix $\alpha$, they can be turned into LMIs based feasibility problem which can be solved via existing software (for example the LMI Control Toolbox of MATLAB).

\section{NUMERICAL EXAMPLE}

Consider the network control systems in the form of (3), where,

$$
\begin{aligned}
\dot{x}(t)= & {\left[\begin{array}{cc}
-1 & 2 \\
0 & -1
\end{array}\right] x(t)+\left[\begin{array}{cc}
-0.1 & 0.2 \\
0 & -0.2
\end{array}\right] x(t-h) } \\
& +\left[\begin{array}{l}
0 \\
1
\end{array}\right] u(t-\tau)+\left[\begin{array}{l}
0.1 \\
0.2
\end{array}\right] \omega(t)
\end{aligned}
$$




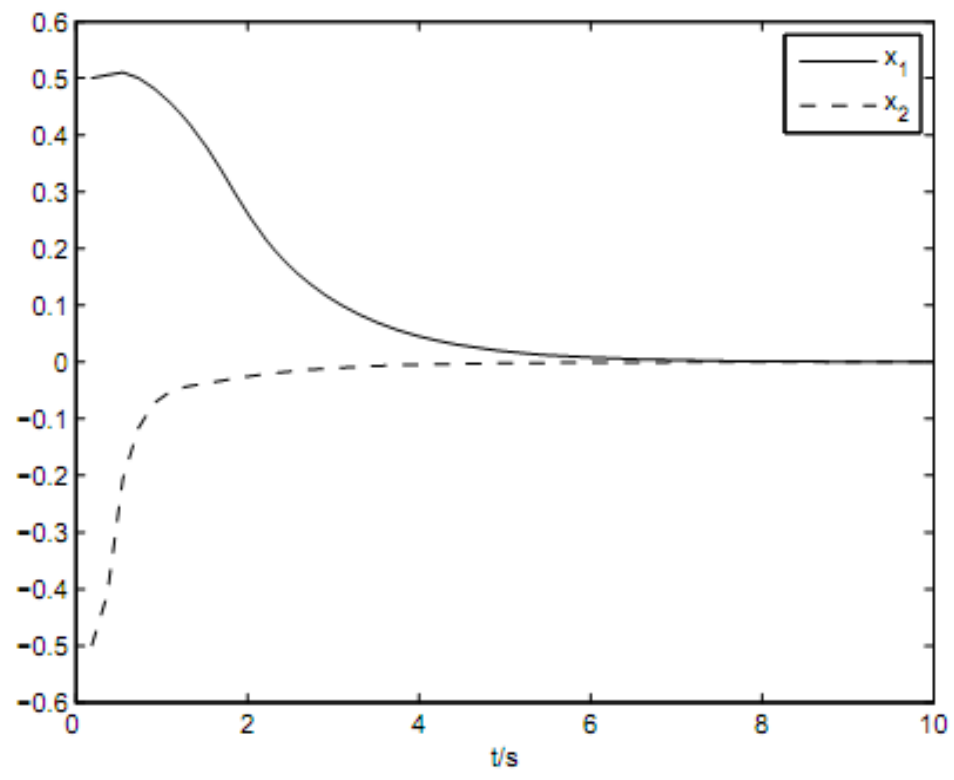

Fig. (2). State $x_{1}(t), x_{2}(t)$ responses of the systems.

In this case, we choose $c_{1}=0.25, \alpha=1, T=2, R=I_{2}$, $d=4, \tau=0.5, h=0.8$. By Solving the LMIs (16-21), we can obtain the controller

$u(t)=\bar{K} X^{-1} x(t)=\left[\begin{array}{ll}-6.5421 & 7.3927\end{array}\right] x(t)$

We select

$x(0)=\left[\begin{array}{c}0.5 \\ -0.5\end{array}\right]$

The simulation result are shown in the following figure.

From the above Fig. (2), it is clear that the finite-time stabilization of NCSs (3) via state feedback of desired closed-loop properties.

\section{CONCLUSION}

In this paper, we considered the finite-time control problem via state feedback for a class of NCSs with state delay and communication delay. Based on finite-time stability theory combined with LMIs techniques, sufficient conditions that ensure the closed-loop system finite-time stability is obtained.

\section{CONFLICT OF INTEREST}

The authors confirm that this article content has no conflicts of interest.

\section{ACKNOWLEDGEMENTS}

The authors wish to thank the editor and the anonymous reviewers for their constructive comments and suggestions to improve the quality and the presentation of the paper. This work is supported by National Nature Science Foundation of China under Grant 61073065; Nature Science Foundation of Henan Province under Grant 092300410145; The Education Department of Henan Province Key Foundation under Grant 13A110023, 14A520003.

\section{REFERENCES}

[1] G.C. Walsh, O. Beldiman, L.G. Bushnell, "Asympto-tic behavior of nonlinear networked control systems", IEEE Transactions on Automatic Control, vol.46, pp.1093-1097, 2001.

[2] G. C. Walsh, H. Ye, L G. Bushnell, "Stability analysis of networked control systems", IEEE Trans. Control Syst Technol, vol.10, pp.438-446, 2002.

[3] S. Hu, Q. Zhu, "Stochastic optimal control and analysis of stability of networked control systems with long delay", Automatica, vol.39, pp.1877-1884, 2003.

[4] D. Yue, Q.L. Han, and J. Lam, "Network-based robust $H$ control of a systems with uncertainty", Automatica, vol.4 pp.999-1007, 2005.

[5] Z. -H. Guan, J. Huang, G. R. Chen, "Stability Analysis of Networked Impulsive Control Systems", Proceeding of 25th Chinese Control Conference, pp.2041-2044, 2006.

[6] Y. Tian, Z. Yu, "Multifractal nature of network induced time delay in networked control systems", Physics Letter A, vol.361, pp.103-107, 2007

[7] D. Dacic, D. Nesic, "Observer design for wired linear networked control systems using matrix inequalities", Automatica, vol.44, pp.2840-2848, 2008.

[8] N. Vatanski, "Networked control with delay measurement and estimation", Control Engineering Practice, vol.17, pp.231-244, 2009.

[9] H. C. Yan, X. H. Huang, M. Wang, H. Zhang, "Delay-dependent stability criteria for a class of networked control systems with multi-input and multi-output", Chaos, Solitons \& Fractals, vol.34, pp.997-1005, 2007.

[10] X. Jiang, Q.-L. Han, S.R. Liu and A. Xue, "A new Hœ stabilization criterion for networked control systems", IEEE Trans. Automat. Control, vol.53, pp.1025-1032, 2008. 
[11] H. Gao, T. Chen, J. Lam, "A new delay system approach to network-based control", Automatica, vol.44, pp.39-52, 2008.

[12] M. W. Hong, C. L. Lin and B. M .Shiu, "Stabilizing network control for pneumatic systems with time-delays", vol.19, pp.399409, 2009.

[13] A. EI-Gohary, "Optimal control of an angular motion of a rigid body during infinite and finite-time intervals", Appl. Math. Comput, vol.141, pp.541-551, 2003.

[14] P. Dorato, "Short time stability in linear time-varying systems", Proceeding of IRE international convention record, vol.4, pp.8387, 1961.

[15] L.Weiss, E. F. Infante, "Finite time stability under perturbing forces and on product spaces", IEEE Trans Automat Control, vol.12, pp.54-59, 1967.

[16] F. Amato, M. Ariola, P. Dorate., "Finite-time control of linear systems subject to parametric uncertainties and disturbances", Automatica, vol.37, pp.1459-1463, 2001.
[17] A. EI-Gohary, A.S. AI-Ruzaiza, "Optimal control of nonhomogenous prey-predator models during infinite and finite-time intervals", Appl. Math. Comput., vol. 146, pp.495-508, 2003.

[18] F. Amato, M. Ariola, "Finite-time control of discrete-time linear system", IEEE Trans Automat Control, vol.50, pp.724-729, 2005.

[19] E. Moulay, W. Perruquetti, "Finite time stability and stabilization of a class of continuous systems", J Math Anal Appl, vol.323, pp.1430-1443, 2006.

[20] F. Amato, M. Ariola, P. Dorate, "Finite-time stabilization via dynamic output feedback", Automatica, vol.42, pp. 337-342, 2006.

[21] E. Moulay, M. Dambrine, N, Yeganefar, W. Perruquetti, "Finitetime stability and stabilization of time-delay systems", Systems Control Letters, vol.57, pp.561-566, 2008.

[22] F. Amato, M. Ariola, C, Cosentino, "Finite-time control of discrete-time linear systems: Analysis and design conditions", $A u$ tomatica, vol.46, pp.919-924, 2010.

Received: September 16, 2014

(C) Hejun Yao; Licensee Bentham Open.

This is an open access article licensed under the terms of the Creative Commons Attribution Non-Commercial License (http://creativecommons.org/licenses/by-nc/3.0/) which permits unrestricted, non-commercial use, distribution and reproduction in any medium, provided the work is properly cited. 